\title{
Profile of brivaracetam and its potential in the treatment of epilepsy
}

\author{
This article was published in the following Dove Press journal: \\ Neuropsychiatric Disease and Treatment \\ 30 November 2015 \\ Number of times this article has been viewed
}

\author{
Edoardo Ferlazzo ${ }^{1,2}$ \\ Emilio Russo ${ }^{3}$ \\ Laura Mumoli' \\ Chiara Sueri² \\ Sara Gasparini ${ }^{1,2}$ \\ Caterina Palleria ${ }^{3}$ \\ Angelo Labate' \\ Antonio Gambardella' \\ Giovambattista De Sarro ${ }^{3}$ \\ Umberto Aguglia ${ }^{1,2}$ \\ 'Department of Medical and Surgical \\ Sciences, Magna Græcia University, \\ Catanzaro, ${ }^{2}$ Regional Epilepsy Centre, \\ Bianchi-Melacrino-Morelli Hospital, \\ Reggio Calabria, ${ }^{3}$ Institute of \\ Pharmacology, Magna Græcia \\ University, Catanzaro, Italy
}

\begin{abstract}
Brivaracetam (BRV) (UCB 34714) is currently under review by the US Food and Drug Administration and European Medicines Agency for approval as an add-on treatment for adult patients with partial seizures. Similar to levetiracetam (LEV), BRV acts as a high-affinity ligand of the synaptic vesicle protein $2 \mathrm{~A}$, however, it has been shown to be 10 - to 30 -fold more potent than LEV. Moreover, BRV does not share the LEV inhibitory activity on the high voltage $\mathrm{Ca}^{2+}$ channels and AMPA receptors, and it has been reported to act as a partial antagonist on neuronal voltage-gated sodium channels. The pharmacokinetic profile of BRV is favorable and linear, and it undergoes an extensive metabolism into inactive compounds, mainly through the hydrolysis of its acetamide group. Furthermore, it does not significantly interact with other antiepileptic drugs and more than $95 \%$ is excreted through the urine, with an unchanged fraction of $8 \%-11 \%$. BRV has a half-life of approximately $8-9$ hours and it is usually given twice daily. To date, a wide range of experimental studies have reported the effectiveness of BRV with regards to partial and generalized seizures. In humans, six randomized, placebo-controlled trials and two meta-analyses highlighted the efficacy, or good tolerability, of BRV as an add-on treatment for patients with uncontrolled partial seizures. A wide dose range of BRV has been evaluated in those trials (5-200 mg), but the most suitable for clinical use appears to be 50-100 mg/day. The most common adverse reactions to BRV are mild to moderate, transient, often improve during the course of the treatment, and mainly consist of central nervous system symptoms, such as fatigue, dizziness, and somnolence. The aim of this paper is to critically review the literature data regarding experimental animal models and clinical trials on BRV, and to define its potential usefulness for the clinicians who manage patients with epilepsy.
\end{abstract}

Keywords: seizures, animal, therapy, drug, antiepileptic

\section{Introduction}

Although the administration of antiepileptic drugs (AEDs) allows freedom from seizures in approximately $75 \%$ of patients with epilepsy, the occurrence of adverse drug reactions (ADR) is still responsible for poor compliance as well as the discontinuation of the therapy in up to $25 \%$ of patients before having reached the effective dose amount. Therefore, this has led to growing costs in clinical management. ${ }^{1,2}$

During the past few decades, research has been focused on the development of AEDs that have a more favorable pharmacological and pharmacodynamic profile. The possibility that antiepileptic efficacy can be reached through the modulation of neuronal hyperexcitability and, in particular, the effect on the synaptic vesicle protein $2 \mathrm{~A}$ $(\mathrm{SV} 2 \mathrm{~A})^{3}$ has recently raised experts' interest. Levetiracetam (LEV) is the first molecule with this target and it represents the ancestor from which several racetam analogs have been subsequently developed. ${ }^{4}$ Brivaracetam (BRV) (2S)-2-([4R]-2-oxo-4-propylpyrrolidinyl)-butanamide is a n-propyl analog of LEV, 5 and its selectivity to SV2A, as well as its potency, have been demonstrated to be greater than those shown by LEV. According
Correspondence: Edoardo Ferlazzo Regional Epilepsy Centre, BianchiMelacrino-Morelli Hospital, Via Melacrino, 89100 Reggio Calabria, Italy Tel +390965 397972

Fax +390965 397973

Email ferlazzo@unicz.it 
to the experimental conditions, its potency for example, can be 10- to 30-fold higher than that of LEV. ${ }^{6,7}$

This article aims to critically review the literature regarding experimental animal models and clinical trials on BRV, and to define its potential usefulness for the clinicians who manage patients with epilepsy.

\section{Methods}

\section{Review of the literature}

References were identified by searching PubMed for articles published prior to October 2015, with the terms "brivaracetam" in combination with "seizures", "epilepsy", and "animal" being searched. Studies were also identified through searches within the authors' own files and one abstract was also included in this revision. The selection criteria included newness, importance, originality, quality, and relevance to the scope of this review.

\section{Statistical analysis}

To describe efficacy and tolerability of BRV across different studies, categorical variables were described as percentages. Differences between groups (BRV and placebo-treated groups) were assessed using Fisher's exact test. The critical $P$-value was set at 0.05 .

\section{Pharmacodynamics of BRV and experiments on animal models of epilepsy}

BRV action on SV2A has been extensively tested. The SV2A protein is involved in neurotransmission by playing a role in the regulation of normal synaptic vesicle cycling and of neurotransmitter release. ${ }^{4,8}$ Even though many aspects of this type of mechanism need further clarification, the association between SV2A binding and an anticonvulsant effect has been demonstrated in experimental studies. ${ }^{8}$

According to preclinical trials on animal models of both partial and generalized epilepsy, BRV demonstrates a higher potency and efficacy when compared to LEV..$^{9,10}$ More precisely, in fully amygdala-kindled rats, BRV can determine a significant decrease in motor-seizure severity at the dosage of $21.2 \mathrm{mg} / \mathrm{kg}$, while LEV shows analogous responses at the dose of $170 \mathrm{mg} / \mathrm{kg}$. Moreover, a greater dose of BRV $(212.3 \mathrm{mg} / \mathrm{kg})$ is responsible for a significant decrease of the after-discharge duration, which is an effect that can be observed with LEV exclusively for dosages of $1,700 \mathrm{mg} / \mathrm{kg}$ or higher. ${ }^{9,10}$ Even in those mice that are resistant to phenytoin (ED50 $68.3 \mathrm{mg} / \mathrm{kg}$, ip), BRV was shown to be effective against both partial and generalized seizures. ${ }^{9,11}$
As can be demonstrated by its acute dosing in audiogenic mice, BRV can cross the blood-brain barrier more easily than LEV. This implies a shorter latency of action, which may prove to be a clinically relevant result in the treatment of emergencies such as status epilepticus or cluster seizures. ${ }^{10}$ Furthermore, Detrait et al reported the effectiveness of BRV on kindled seizures that are relevantly modulated for seizure threshold and severity. ${ }^{12}$

In a work evaluating the rapid kindling model in P14, P21, P28, and P60 rats, a $100 \mathrm{mg} / \mathrm{kg}$ dose of BRV succeeded in determining a significant increase in the after-discharge threshold at all ages, while the same drug at the dosage of $10 \mathrm{mg} / \mathrm{kg}$ was effective in P60, P28, and P21 rats only. ${ }^{13}$ Data regarding the reduction of the after-discharge duration indicate that 10 and $100 \mathrm{mg} / \mathrm{kg}$ doses of BRV are significantly effective at P60, whereas only the $100 \mathrm{mg} / \mathrm{kg}$ dose is effective at P21. At P60, the administration of BRV implies a higher number of stimulations to reach stage 4-5 seizures in a dose-dependent manner. Similarly, at P28 and P21, the administration of BRV determines the almost complete disappearance of stage 4-5 seizures, and requires an increased number of stimulations to evoke stage 4-5 seizures in a dose-dependent modality. ${ }^{13}$

Furthermore, in a model of self-sustained status epilepticus, evoked by the stimulation of the perforant path, BRV demonstrated a synergic action with diazepam in decreasing the seizures' duration. ${ }^{14}$ In agreement to such data, BRV (0.3 mg/kg) has recently been shown to have higher anti-seizure and antimyoclonic activity, when compared to LEV (3 mg/ $\mathrm{kg})$ in an animal model of post-hypoxic myoclonus. ${ }^{15}$

Noticeably, growing evidence indicates that in transgenic Alzheimer's disease mice, BRV may not only play a role similar to that of ethosuximide with regards to spike-wave discharges, but it may also be effective in contrasting memory deterioration. Therefore, this new AED potentially shows an extended profile of action. ${ }^{16}$

Additionally to its role in the SV2A blockage, BRV shares with other AEDs a potential, but still debated inhibitory effect on neuronal voltage-gated sodium channels, by acting as a partial antagonist. ${ }^{17-19}$

\section{Pharmacokinetics of BRV}

The pharmacokinetic profile of BRV has been evaluated in healthy adult volunteers, the elderly, patients with epilepsy, and patients with hepatic or renal dysfunction..$^{20-24}$ The drug exhibits favorable pharmacokinetic properties due to its linear and predictable profile, with low inter-subject variability and close to $100 \%$ bioavailability. ${ }^{11,20-22}$ The pharmacokinetic 
differences in elderly patients, as compared to healthy volunteers, are not relevant and therefore dose adjustment does not seem to be required. ${ }^{24}$

Following oral administration, BRV is quickly absorbed at a gastrointestinal level with a linear and dose-dependent profile, and it is unaffected by the presence of food. ${ }^{21,25}$

The distribution volume of BRV is close to the total body water content $(\mathrm{Vz}=0.5 \mathrm{~L} / \mathrm{kg})$ and it is weakly bound $(17.5 \%)$ to plasma proteins. Terminal half-life is approximately $8-9$ hours ${ }^{20}$ and BRV is usually administered twice daily in equal doses.

BRV undergoes an extensive metabolism into three pharmacologically inactive compounds (UCB data files) and more than $95 \%$ is excreted through the urine with an unchanged fraction of $8 \%-11 \% .{ }^{11,20}$ The main metabolic pathway consists of the hydrolysis of BRV's acetamide group, leading to the formation of an acid metabolite (BRV-AC; $34.2 \%$ of the radiolabeled urinary dose) ${ }^{20} \mathrm{~A}$ smaller proportion of the drug is converted by the cytochrome P450 $2 \mathrm{C} 19^{26}$ into a hydroxy metabolite (BRV-OH; $15.9 \%$ of the urinary dose). ${ }^{20}$ From the participation of both of these pathways, a hydroxyacid metabolite is produced $(15.2 \%$ of the dose in the urine).$^{20}$
An open-label trial on patients with liver dysfunction revealed that the plasmatic half-life of BRV may increase up to 17.4 hours, depending on the severity of the hepatic disease. ${ }^{24}$ Nevertheless, the exposure to BRV increases by $50 \%-60 \%$ in patients with hepatic impairment, irrespective of the severity of the pathology, defined on the basis of the Child-Pugh score. ${ }^{24,25}$ Thus, according to such evidence, the maximum daily dose of BRV should be reduced by one-third in patients with liver disease. ${ }^{24}$

When exposed to a single $200 \mathrm{mg}$ oral dose of BRV, patients with severe kidney dysfunction not requiring dialysis (creatinine clearance $<15 \mathrm{~mL} / \mathrm{min}$ ) show a 10 -fold decrement on renal excretion of the three metabolites, but it is not clear if adjustment of the dose is required. ${ }^{23}$

\section{Efficacy of BRV Effect of BRV on partial seizures}

Literature data assessing the efficacy and tolerability of BRV as an adjunctive treatment in patients with drug-resistant partial seizures, consist of six randomized, placebo-controlled, clinical trials (Table 1) ) $^{27-32}$ and two meta-analyses. ${ }^{33,34}$

In the study by French et al (Table 1), ${ }^{27}$ the efficacy (in terms of median percentage reduction over placebo from the baseline of seizure frequency per week) was related to the

Table I Six randomized, placebo-controlled trials of adjunctive BRV in patients with uncontrolled seizures

\begin{tabular}{|c|c|c|c|}
\hline Trial & $\begin{array}{l}\text { ITT population } \\
\text { (seizure type) }\end{array}$ & $\begin{array}{l}\text { Brivaracetam } \\
\text { range }\end{array}$ & Responder rates $\geq \mathbf{5 0} \%$ for $\mathrm{PBO}$ and $\mathrm{BRV}$ \\
\hline \multirow[t]{4}{*}{ French et $\mathrm{al}^{27}$} & BRV I54 & $5-50 \mathrm{mg} / \mathrm{day}$ & $16.7 \%$ for $\mathrm{PBO}$ \\
\hline & PBO 54 & & $32.0 \%$ for BRV 5 mg/day* \\
\hline & (partial) & & $44.2 \%$ for BRV $20 \mathrm{mg} /$ day* \\
\hline & & & $55.8 \%$ for BRV $50 \mathrm{mg} /$ day* \\
\hline \multirow[t]{3}{*}{ Van Paesschen et $\mathrm{al}^{28}$} & BRV I05 & $50-150 \mathrm{mg} /$ day & $17.3 \%$ for $\mathrm{PBO}$ \\
\hline & PBO 52 & & $35.8 \%$ for BRV 50 mg/day* \\
\hline & (partial) & & $30.8 \%$ for BRV $150 \mathrm{mg} / \mathrm{day}$ \\
\hline \multirow[t]{4}{*}{ Ryvlin et $\mathrm{a}^{29}$} & BRV 298 & $20-100 \mathrm{mg} /$ day & $20.0 \%$ for $\mathrm{PBO}$ \\
\hline & PBO 100 & & $27.3 \%$ for BRV $20 \mathrm{mg} /$ day \\
\hline & (partial) & & $27.3 \%$ for BRV $50 \mathrm{mg} /$ day \\
\hline & & & $36.0 \%$ for BRV $100 \mathrm{mg} /$ day* \\
\hline \multirow[t]{4}{*}{ Biton et $\mathrm{al}^{30}$} & BRV 298 & $5-50 \mathrm{mg} / \mathrm{day}$ & $16.7 \%$ for $\mathrm{PBO}$ \\
\hline & PBO 98 & & $21.9 \%$ for BRV $5 \mathrm{mg} /$ day \\
\hline & (partial) & & $23.2 \%$ for BRV $20 \mathrm{mg} /$ day \\
\hline & & & $32.7 \%$ for BRV $50 \mathrm{mg} /$ day* \\
\hline \multirow[t]{4}{*}{ Kwan et $\mathrm{a}^{31}$} & BRV 359 (323 partial; & Flexible doses & $16.7 \%$ for $\mathrm{PBO}$ \\
\hline & 36 generalized) & $20-150 \mathrm{mg} /$ day & $30.3 \%$ in BRV group with partial seizures* \\
\hline & PBO I2I ( 108 focal; & & $15.4 \%$ for $\mathrm{PBO}$ \\
\hline & 13 generalized) & & $44.4 \%$ in BRV group with generalized seizures \\
\hline \multirow[t]{3}{*}{ Klein et $\mathrm{al}^{32}$} & BRV 50I & $100-200 \mathrm{mg} /$ day & $21.6 \%$ for $\mathrm{PBO}$ \\
\hline & PBO 259 & & $38.9 \%$ for BRV 100 mg/day* \\
\hline & (partial) & & $37.8 \%$ for BRV $200 \mathrm{mg} /$ day* \\
\hline
\end{tabular}

Notes: *Significantly different $(P<0.05)$ from placebo. Copyright @2015. Dove Medical Press. Adapted from Mumoli L, Palleria $C$, Gasparini S, et al. Brivaracetam: review of its pharmacology and potential use as adjunctive therapy in patients with partial onset seizures. Drug Des Devel Ther. 2015;9:5719-5725."

Abbreviations: BRV, brivaracetam; ITT, intention-to-treat; NR, not reported data; PBO, placebo. 
administered dose of BRV (ranging from 5-50 mg/day), but was statistically significant only for BRV $50 \mathrm{mg} /$ day $(9.8 \%$ for BRV $5 \mathrm{mg} /$ day [ $P=0.240]$; 14.9\% for BRV $20 \mathrm{mg} /$ day $[P=0.062] ; 22.1 \%$ for BRV $50 \mathrm{mg} /$ day $[P=0.004])$. Seizure freedom rates during an observational treatment period of 7 weeks were $1.9 \%$ with placebo, $8.0 \%$ with BRV $5 \mathrm{mg} /$ day, $7.7 \%$ with BRV $20 \mathrm{mg} /$ day, and $7.7 \%$ with BRV at $50 \mathrm{mg} /$ day.

In the Van Paesschen et al trial (Table 1$),{ }^{28}$ primary efficacy analysis did not reach statistical significance. In particular, the percent reduction in baseline-adjusted partial seizure frequency/week over placebo during the 7-week maintenance period over placebo was not significant in those treated with BRV $50 \mathrm{mg} /$ day $(14.7 \%$ reduction, $P=0.093)$, or with BRV $150 \mathrm{mg} /$ day $(13.6 \%$ reduction, $P=0.124) .{ }^{28}$ However, a significant difference over placebo for the same outcome measure was reported during the 10-week total treatment period for the BRV $50 \mathrm{mg} /$ day group $(17.7 \%$ reduction, $P=0.026)$, as well as for the BRV $150 \mathrm{mg} /$ day group $(16.3 \%$ reduction, $P=0.043)$. Seizure freedom was reached by a total of nine patients (five patients [9.4\%] with BRV $50 \mathrm{mg} /$ day; three patients [5.8\%] with BRV $150 \mathrm{mg} /$ day; and one patient [1.9\%] with placebo). ${ }^{28}$

$\mathrm{BRV}$ at a daily dose of $100 \mathrm{mg}$ /day showed efficacy in the trial by Ryvlin et al (Table 1). ${ }^{29}$ The percentage reduction over placebo in the baseline-adjusted seizure frequency per week was $6.8 \%$ in those treated with BRV $20 \mathrm{mg} /$ day $(P=0.239)$, $6.5 \%$ in those treated with BRV $50 \mathrm{mg} /$ day $(P=0.261)$, and $11.7 \%$ in those treated with BRV $100 \mathrm{mg} /$ day $(P=0.037)$. Seizure freedom was achieved by two $(2 \%)$ patients treated with BRV $20 \mathrm{mg} /$ day, no patients who were treated with BRV $50 \mathrm{mg} /$ day, four (4\%) patients treated with BRV $100 \mathrm{mg} /$ day, and no patients who were treated with the placebo. ${ }^{29}$

The trial by Biton et al highlighted that BRV has a doserelated efficacy. ${ }^{30}$ In particular, the median reduction in the percentage over placebo from the baseline of seizure frequency per week was $-0.9 \%(P=0.885)$ for BRV $5 \mathrm{mg} / \mathrm{day}$, 4.1\% $(P=0.492)$ for BRV $20 \mathrm{mg} / \mathrm{day}$, and $12.8 \%(P=0.025)$ for BRV $50 \mathrm{mg} /$ day. $^{30}$ Seizure freedom was reached by a total of six patients (no patients treated with the placebo, one $[1.1 \%$ ] patient treated with BRV $5 \mathrm{mg} /$ day, one [1.1\%] patient treated with in BRV $20 \mathrm{mg} / \mathrm{day}$, and four [4.0\%] patients treated with BRV $50 \mathrm{mg} /$ day).

The trial carried out by Kwan et $\mathrm{al}^{31}$ (Table 1) tested BRV at individually-tailored doses ranging from 20-150 mg/day in patients suffering from either partial or generalized refractory seizures. ${ }^{31}$ The baseline-adjusted percentage reduction over placebo of the partial seizure frequency per week was not significant in patients with partial seizures $(7.3 \% ; P=0.125) .{ }^{31}$ Five $(1.5 \%)$ patients with partial seizures were seizure free during the treatment period.

The trial by Klein et al $^{32}$ (Table 1) showed a significant efficacy of BRV at 100 and $200 \mathrm{mg} /$ day, as compared to the placebo with a median percentage decrement over placebo in a 28-day adjusted seizure frequency of $22.8 \%$ when using BRV $100 \mathrm{mg} /$ day $(P<0.001)$, and of $23.2 \%$ when using BRV $200 \mathrm{mg} /$ day $(P<0.001) .{ }^{32}$ During the treatment period, 25 patients reached seizure freedom (two [0.8\%] patients treated with placebo; 13 [5.2\%] patients treated with BRV $100 \mathrm{mg} /$ day $[P=0.003$ compared to placebo]; and ten $[4.0 \%]$ patients treated with BRV $200 \mathrm{mg} /$ day $[P=0.019$ compared to placebo]).

In five of these trials ${ }^{27-31}$ the continuation of LEV as allowed. It seems that patients with a concomitant use of LEV did not respond as well to BRV in comparison to the patients not currently taking LEV, suggesting that its concomitant use may decrease BRV efficacy. Due to the small number of patients taking LEV in these trials, further studies should be performed to evaluate this pharmacodynamic interaction. ${ }^{33}$

\section{Effect of BRV on generalized seizures}

In analogy with LEV, BRV may find an indication for generalized epilepsies. ${ }^{35,36}$

The only trial evaluating the efficacy of BRV on generalized seizures was conducted by Kwan et al. ${ }^{31}$ In this study, 49 patients had generalized seizures, mostly tonicclonic (30 patients), absences (14 patients), and myoclonic (14 patients). Two (5.6\%) patients with generalized seizures were seizure free during the treatment period. The number of generalized seizure days per week reduced from 1.42 at baseline to 0.63 in the BRV-treated patients $(n=36)$, and from 1.47 at baseline to 1.26 in the placebo group $(n=13){ }^{31}$ Moreover, the median percentage reduction from baseline in generalized seizure days per week was $42.6 \%$ in the BRV group versus $20.7 \%$ in the placebo group (statistical significance not shown).

In a Phase IIA, single blind, placebo-controlled study evaluating 18 patients with idiopathic generalized epilepsies and photosensitivity, BRV was found to have the ability to suppress generalized photoparoxysmal responses (PPR) on electroencephalogram. ${ }^{37}$ No patients achieved PPR abolishment with the placebo, whereas 14 (78\%) patients experienced complete abolishment with BRV in this study. ${ }^{37}$ Among the evaluated dosages $(10,20,40$, or $80 \mathrm{mg} /$ day $), 80 \mathrm{mg}$ was the most effective, resulting in long-lasting abolishment of the PPR. ${ }^{37}$ 
Further studies are needed to confirm the efficacy of BRV in patients with generalized seizures.

\section{Effect of BRV in progressive myoclonic epilepsies}

$\mathrm{BRV}$, as compared to LEV, may have potential in the management of progressive myoclonic epilepsies. ${ }^{38,39}$ However, two randomized, placebo-controlled trials assessing the efficacy and tolerability of BRV (5-150 mg/day) as an add-on therapy in Unverricht-Lundborg disease (the most frequent and less severe form of progressive myoclonic epilepsies), ${ }^{40,41}$ did not report a significant improvement of myoclonus. ${ }^{42}$ These trials had some limitations, especially the small sample size and the unpredictable inter- and intra-subject variability of myoclonus in Unverricht-Lundborg disease. Moreover, patients were allowed to receive LEV. ${ }^{42}$ Due to these reasons, further studies evaluating BRV in progressive myoclonic epilepsies are needed.

\section{Safety profile of BRV}

BRV has been shown to have a favorable safety profile, as the side effects reported in association with its administration are mild to moderate and usually do not affect the compliance to the treatment (Table 2). In actuality, a dose of $20-150 \mathrm{mg} /$ day has shown to be well tolerated, with a discontinuation rate of $6.1 \%$ due to ADRs, in comparison to the $5.0 \%$ rate reported in the placebo group. ${ }^{31}$ The tolerability of BRV is even more impressive when considering that its side effects appear to be transient and to decrease in intensity during the course of treatment. In adult patients, the main ADRs are represented by symptoms of the central nervous system consisting of sedation, fatigue, and dizziness (Table 2). ${ }^{21}$ The severity of the somnolence induced by BRV as evaluated by psychometric tests is dose-related, becoming clear for a dosage $\geq 600 \mathrm{mg}$ with symptoms such as decreased attention, motor control, and alertness manifesting. ${ }^{21}$ The type and the intensity of ADRs is not affected by food intake. ${ }^{21}$ Even when prescribed at very high doses (up to $800 \mathrm{mg} /$ day), BRV does not affect cardiac function. ${ }^{43}$

Twice-daily administration of BRV reduces the peak-totrough fluctuations of plasma concentrations and minimizes the adverse reactions, as observed in healthy males. ${ }^{21}$

Experimental studies on animals revealed the absence of any side effects of BRV on fertility and pregnancy (BRV at $400 \mathrm{mg} / \mathrm{kg} /$ day), or fetal development (BRV at $600 \mathrm{mg} /$ day). ${ }^{25}$ However, the effects of treatment with BRV on fertility, or its eventual teratogenicity, are still to be evaluated in humans.

The aforementioned six randomized, placebo-controlled clinical trials included 2,403 patients recruited by intentionto-treat analysis (1,715 patients treated with BRV and 688 patients treated with placebo). ${ }^{27-32}$ The percentage of patients complaining of at least one side effect was significantly higher in those taking BRV $(66.1 \%$ in the BRV group and $60.5 \%$ in

Table 2 Most common adverse effects of BRV in six randomized, placebo-controlled clinical trials

\begin{tabular}{|c|c|c|c|c|c|}
\hline Trial & Headache & Somnolence & Fatigue & Dizziness & $\begin{array}{l}\text { Gastrointestinal } \\
\text { disturbance }\end{array}$ \\
\hline \multirow[t]{4}{*}{ French et $\mathrm{al}^{27}$} & BRV 5: 8\% & BRV 5: $2 \%$ & BRV 5: $0 \%$ & BRV 5: $2 \%$ & NR \\
\hline & BRV $20: 3.8 \%$ & BRV 20: $5.8 \%$ & BRV $20: 3.8 \%$ & BRV 20: 0\% & \\
\hline & BRV 50: $1.9 \%$ & BRV 50: 5.8\% & BRV 50: $5.8 \%$ & BRV 50: 7.7\% & \\
\hline & PBO: $7.4 \%$ & PBO: $7.4 \%$ & PBO: $3.7 \%$ & PBO: $5.6 \%$ & \\
\hline \multirow[t]{3}{*}{ Van Paesschen et $\mathrm{al}^{28}$} & BRV 50: $15.1 \%$ & BRV 50: $9.4 \%$ & BRV 50: $13.2 \%$ & BRV 50: $3.8 \%$ & BRV 50: $9.4 \%$ \\
\hline & BRV I50: 7.7\% & BRV I50: $5.8 \%$ & BRV I50: 5.8\% & BRV I50: $9.6 \%$ & BRV I50: $19.3 \%$ \\
\hline & PBO: $7.7 \%$ & PBO: $5.8 \%$ & PBO: $7.7 \%$ & PBO: $5.8 \%$ & PBO: $15.3 \%$ \\
\hline \multirow[t]{4}{*}{ Ryvlin et $\mathrm{al}^{29}$} & BRV 20: $14.1 \%$ & BRV 20: $8.1 \%$ & BRV 20: 3\% & BRV 20: $5.1 \%$ & BRV 20: 0\% \\
\hline & BRV 50: $18.2 \%$ & BRV 50: $6.1 \%$ & BRV 50: 4\% & BRV 50: $7.1 \%$ & BRV 50: I\% \\
\hline & BRV I00: $9 \%$ & BRV I00: 8\% & BRV I00: $8 \%$ & BRV I00: $5 \%$ & BRV I00: $6 \%$ \\
\hline & PBO: $9 \%$ & PBO: $6 \%$ & PBO: $2 \%$ & PBO: $8 \%$ & PBO: $4 \%$ \\
\hline \multirow[t]{4}{*}{ Biton et $\mathrm{al}^{30}$} & BRV 5: II.3\% & BRV 5: $14.4 \%$ & BRV 5: 3.1\% & BRV 5: $12.4 \%$ & BRV 5: II.3\% \\
\hline & BRV 20: $6 \%$ & BRV 20: $14 \%$ & BRV 20: $13 \%$ & BRV 20: $14 \%$ & BRV 20: $12 \%$ \\
\hline & BRV 50: $13 \%$ & BRV 50: $16.8 \%$ & BRV 50: $9.9 \%$ & BRV 50: $15.8 \%$ & BRV 50: $16.8 \%$ \\
\hline & PBO: $14.3 \%$ & PBO: $7.1 \%$ & PBO: $2 \%$ & PBO: $9.2 \%$ & PBO: $6.1 \%$ \\
\hline \multirow[t]{2}{*}{ Kwan et al ${ }^{31}$} & BRV: $14.2 \%$ & BRV: $14.1 \%$ & BRV: $7.8 \%$ & BRV: $8.6 \%$ & BRV: $5.6 \%$ \\
\hline & PBO: $19.8 \%$ & PBO: $4.1 \%$ & PBO: $4.1 \%$ & PBO: $5.8 \%$ & PBO: $8.3 \%$ \\
\hline \multirow[t]{3}{*}{ Klein et $\mathrm{al}^{32}$} & BRV I00: $6.7 \%$ & BRV I00: $19.4 \%$ & BRV I00: 7.5\% & BRV I00: $10.3 \%$ & NR \\
\hline & BRV 200: $8.0 \%$ & BRV 200: $16.8 \%$ & BRV 200: I I.6\% & BRV 200: $14.4 \%$ & \\
\hline & PBO: $8.4 \%$ & PBO: $7.7 \%$ & PBO: $3.8 \%$ & PBO: $5 \%$ & \\
\hline
\end{tabular}

Notes: Data from these studies. ${ }^{27-32}$ Dose for BRV is $\mathrm{mg} / \mathrm{day}$

Abbreviations: BRV, brivaracetam; NR, not reported; PBO, placebo. 
the placebo group, $P=0.008)$. However, the majority of the reactions were mild or moderate, and the discontinuation rate, secondary to adverse effects, was very low and similar in both groups $(6.0 \%$ in the BRV group and $4.0 \%$ in the placebo group, $P=0.06)$. Serious ADRs were rarely observed in a similar proportion in both groups $(2.9 \%$ in patients treated with BRV and $4.4 \%$ in patients treated with placebo, $P=0.20$ ). The following single ADRs were observed to be significantly more frequent in patients treated with BRV, compared to those patients on the placebo; dizziness ( $10 \%$ vs $6.3 \%, P=0.002)$, fatigue $(7.7 \%$ vs $3.6 \%, P<0.001)$, and somnolence ( $12 \%$ vs $6.6 \%, P<0.001)$. Irritability was documented in only three trials among a small percentage of patients $(3 \%$ in the BRV group and $1 \%$ in the placebo group, $P=0.36$ ).

The eventual negative side effects on sexual functioning, described for LEV as well as different new AEDs, ${ }^{44-46}$ also need to be investigated for BRV.

\section{Drug interactions}

Therapy with BRV does not significantly affect the plasma concentrations of other AEDs. ${ }^{47}$ Nevertheless, carbamazepine blood levels appear to be slightly decreased in case of concurrent intake of BRV (400 mg/day). At the same time, the blood concentration of carbamazepine-epoxide increases in a dosedependent way ${ }^{25,48}$ as a result of the negative influence of BRV on epoxide hydrolase, which is the enzyme responsible for the conversion of carbamazepine-epoxide into carbamazepinediol. ${ }^{48}$ While therapeutic doses of BRV (100 mg/day) do not affect oral contraceptive efficacy, high doses of BRV (400 mg/ day) may slightly decrease serum levels of ethinylestradiol and levonorgestrel, without any impact on ovulation. ${ }^{49}$

\section{Conclusion}

BRV represents a new generation of AED, with an action partially overlapping with that of LEV, and a possible inhibitory influence on voltage-gated sodium channels which is a mechanism that is also observed in several other AEDs. ${ }^{50} \mathrm{On}$ this basis, it is possible to presume that BRV is at least as effective as LEV, indicating that it is a reasonable adjunctive therapy in patients with partial epilepsy. The efficacy or tolerability of BRV on drug-resistant partial seizures has actually been evaluated and confirmed by six randomized, placebocontrolled trials ${ }^{27-32}$ and by two recent meta-analyses. ${ }^{33,34}$ A wide range of BRV dosages has been evaluated in these trials (5-200 mg/day), but the most suitable for clinical use appears to be 50-100 mg/day. Further studies are needed to evaluate BRV efficacy in generalized seizures and to investigate the long-term efficacy and safety of this drug.

\section{Disclosure}

E Ferlazzo received speaker fees from UCB. E Russo received speaker fees from Almirall, Eisai, and Lundbeck. A Gambardella received speaker fees from UCB, Eisai, and Novartis. A Labate received speaker fees from UCB. The authors report no other conflicts of interest in this work.

\section{References}

1. Perucca P, Gilliam FG. Adverse effects of antiepileptic drugs. Lancet Neurol. 2012;11(9):792-802.

2. Leporini C, De Sarro G, Russo E. Adherence to therapy and adverse drug reactions: is there a link? Expert Opin Drug Saf. 2014;13 Suppl 1: S41-S55.

3. Lyseng-Williamson KA. Levetiracetam: a review of its use in epilepsy. Drugs. 2011;71(4):489-514.

4. Mendoza-Torreblanca JG, Vanoye-Carlo A, Phillips-Farfán BV, CarmonaAparicio L, Gómez-Lira G. Synaptic vesicle protein 2A: basic facts and role in synaptic function. Eur J Neurosci. 2013;38(11):3529-3539.

5. Kenda BM, Matagne AC, Talaga PE, et al. Discovery of 4-substituted pyrrolidone butanamides as new agents with significant antiepileptic activity. J Med Chem. 2004;47(3):530-549.

6. Lynch BA, Lambeng N, Nocka K, et al. The synaptic vesicle protein SV2A is the binding site for the antiepileptic drug levetiracetam. Proc Natl Acad Sci U S A. 2004;101(26):9861-9866.

7. Gillard M, Fuks B, Leclercq K, Matagne A. Binding characteristics of brivaracetam, a selective, high affinity SV2A ligand in rat, mouse and human brain: relationship to anti-convulsant properties. Eur J Pharmacol. 2011;664(1-3):36-44.

8. Kaminski RM, Matagne A, Leclercq K, et al. SV2A protein is a broadspectrum anticonvulsant target: functional correlation between protein binding and seizure protection in models of both partial and generalized epilepsy. Neuropharmacology. 2008;54(4):715-720.

9. Matagne A, Margineanu DG, Kenda B, Michel P, Klitgaard H. Anti-convulsive and anti-epileptic properties of brivaracetam (ucb 34714), a high-affinity ligand for the synaptic vesicle protein, SV2A. Br J Pharmacol. 2008;154(8):1662-1671.

10. Bialer M, Johannessen SI, Levy RH, Perucca E, Tomson T, White HS. Progress report on new antiepileptic drugs: a summary of the Twelfth Eilat Conference (EILAT XII). Epilepsy Res. 2015;111:85-141.

11. Mumoli L, Palleria C, Gasparini S, et al. Brivaracetam: review of its pharmacology and potential use as adjunctive therapy in patients with partial onset seizures. Drug Des Devel Ther. 2015;9:5719-5725.

12. Detrait ER, Leclercq K, Loscher W, et al. Brivaracetam does not alter spatial learning and memory in both normal and amygdala-kindled rats. Epilepsy Res. 2010;91(1):74-83.

13. Dupuis N, Matagne A, Staelens L, et al. Anti-ictogenic and antiepileptogenic properties of brivaracetam in mature and immature rats. Epilepsia. 2015;56(5):800-805.

14. Wasterlain CG, Baldwin R, Naylor DE, Thompson KW, Suchomelova L, Niquet J. Rational polytherapy in the treatment of acute seizures and status epilepticus. Epilepsia. 2011;52 Suppl 8:70-71.

15. Tai KK, Truong DD. Brivaracetam is superior to levetiracetam in a rat model of post-hypoxic myoclonus. J Neural Transm (Vienna). 2007;114(12):1547-1551

16. Nygaard HB, Kaufman AC, Sekine-Konno T, et al. Brivaracetam, but not ethosuximide, reverses memory impairments in an Alzheimer's disease mouse model. Alzheimers Res Ther. 2015;7(1):25.

17. Zona C, Pieri M, Carunchio I, Curcio L, Klitgaard H, Margineanu DG. Brivaracetam (ucb 34714) inhibits $\mathrm{Na}(+$ ) current in rat cortical neurons in culture. Epilepsy Res. 2010;88(1):46-54.

18. Köhling R. Voltage-gated sodium channels in epilepsy. Epilepsia. 2002; 43(11):1278-1295.

19. Rogawski MA, Löscher W. The neurobiology of antiepileptic drugs. Nature Reviews Neuroscience. 2004;5(7):553-564. 
20. Sargentini-Maier ML, Espie P, Coquette A, Stockis A. Pharmacokinetics and metabolism of 14C-brivaracetam, a novel SV2A ligand, in healthy subjects. Drug Metab Dispos. 2008;36(1):36-45.

21. Sargentini-Maier ML, Rolan P, Connell J, et al. The pharmacokinetics, CNS pharmacodynamics and adverse event profile of brivaracetam after single increasing oral doses in healthy males. Br J Clin Pharmacol. 2007;63(6):680-688.

22. Rolan P, Sargentini-Maier ML, Pigeolet E, Stockis A. The pharmacokinetics, CNS pharmacodynamics and adverse event profile of brivaracetam after multiple increasing oral doses in healthy men. $\mathrm{Br} J$ Clin Pharmacol. 2008;66(1):71-75.

23. Sargentini-Maier ML, Sokalski A, Boulanger P, Jacobs T, Stockis A Brivaracetam disposition in renal impairment. J Clin Pharmacol. 2012;52(12):1927-1933.

24. Stockis A, Sargentini-Maier ML, Horsmans Y. Brivaracetam disposition in mild to severe hepatic impairment. J Clin Pharmacol. 2013;53(6): 633-641.

25. von Rosenstiel P. Brivaracetam (UCB 34714). Neurotherapeutics. 2007;4(1):84-87.

26. Stockis A, Watanabe S, Rouits E, Matsuguma K, Irie S. Brivaracetam single and multiple rising oral dose study in healthy Japanese participants: influence of CYP2C19 genotype. Drug Metab Pharmacokinet. 2014;29(5):394-399.

27. French JA, Costantini C, Brodsky A, von Rosenstiel P, Group NS Adjunctive brivaracetam for refractory partial-onset seizures: a randomized, controlled trial. Neurology. 2010;75(6):519-525.

28. Van Paesschen W, Hirsch E, Johnson M, Falter U, von Rosenstiel P. Efficacy and tolerability of adjunctive brivaracetam in adults with uncontrolled partial-onset seizures: a phase IIb, randomized, controlled trial. Epilepsia. 2013;54(1):89-97.

29. Ryvlin P, Werhahn KJ, Blaszczyk B, Johnson ME, Lu S. Adjunctive brivaracetam in adults with uncontrolled focal epilepsy: results from a double-blind, randomized, placebo-controlled trial. Epilepsia. 2014;55(1):47-56

30. Biton V, Berkovic SF, Abou-Khalil B, Sperling MR, Johnson ME, Lu S. Brivaracetam as adjunctive treatment for uncontrolled partial epilepsy in adults: a phase III randomized, double-blind, placebocontrolled trial. Epilepsia. 2014;55(1):57-66.

31. Kwan P, Trinka E, Van Paesschen W, Rektor I, Johnson ME, Lu S Adjunctive brivaracetam for uncontrolled focal and generalized epilepsies: results of a phase III, double-blind, randomized, placebocontrolled, flexible-dose trial. Epilepsia. 2014;55(1):38-46.

32. Klein P, Schiemann J, Sperling MR, et al. A randomized, double-blind, placebo-controlled, multicenter, parallel-group study to evaluate the efficacy and safety of adjunctive brivaracetam in adult patients with uncontrolled partial-onset seizures. Epilepsia. Epub 2015 Oct 16.

33. Tian X, Yuan M, Zhou Q, Wang X. The efficacy and safety of brivaracetam at different doses for partial-onset epilepsy: a meta-analysis of placebo-controlled studies. Expert Opinion on Pharmacotherapy. 2015;16(12):1755-1767.
34. Ma J, Huang S, You C. Adjunctive brivaracetam for patients with refractory partial seizures: a meta-analysis of randomized placebocontrolled trials. Epilepsy Res. 2015;114:59-65.

35. Crespel A, Gelisse P, Reed RC, et al. Management of juvenile myoclonic epilepsy. Epilepsy Behav. 2013;28 Suppl 1:S81-S86.

36. Kasteleijn-Nolst Trenité DG, Schmitz B, Janz D, et al. Consensus on diagnosis and management of JME: from founder's observations to current trends. Epilepsy Behav. 2013;28 Suppl 1:S87-S90.

37. Kasteleijn-Nolst Trenité DG, Genton P, Parain D, et al. Evaluation of brivaracetam, a novel SV2A ligand, in the photosensitivity model. Neurology. 2007;69(10):1027-1034.

38. Magaudda A, Gelisse P, Genton P. Antimyoclonic effect of levetiracetam in 13 patients with Unverricht-Lundborg disease: clinical observations. Epilepsia. 2004;45(6):678-681.

39. Muona M, Berkovic SF, Dibbens LM, et al. A recurrent de novo mutation in $\mathrm{KCNC} 1$ causes progressive myoclonus epilepsy. Nat Genet. 2015;47(1):39-46.

40. Magaudda A, Ferlazzo E, Nguyen VH, Genton P. Unverricht-Lundborg disease, a condition with self-limited progression: long-term follow-up of 20 patients. Epilepsia. 2006;47(5):860-866.

41. Franceschetti S, Michelucci R, Canafoglia L, et al. Progressive myoclonic epilepsies: definitive and still undetermined causes. Neurology. 2014;82(5):405-411.

42. Kälviäinen G, Genton P, Andermann E, et al. Brivaracetm in patients with Unverricht-Lundborg Disease: results from two randomized, placebo-controlled, double-blind studies. In: Epilepsia Conference Proceedings: 28th International Epilepsy Congress; June 28-July 2, 2009; Budapest. Abstract.

43. Rosillon D, Astruc B, Hulhoven R, et al. Effect of brivaracetam on cardiac repolarisation - a thorough QT study. Curr Med Res Opin. 2008;24(8): 2327-2337.

44. Calabrò RS, Italiano D, Militi D, Bramanti P. Levetiracetam-associated loss of libido and anhedonia. Epilepsy Behav. 2012;24(2):283-284.

45. Calabrò RS, Ferlazzo E, Italiano D, Bramanti P. Dose-dependent oxcarbazepine-related anorgasmia. Epilepsy Behav. 2010;17(2): 287-288.

46. Calabrò RS, Bramanti P, Italiano D, Ferlazzo E. Topiramate-induced erectile dysfunction. Epilepsy Behav. 2009;14(3):560-561.

47. Bialer M, Johannessen SI, Levy RH, Perucca E, Tomson T, White HS Progress report on new antiepileptic drugs: a summary of the Ninth Eilat Conference (EILAT IX). Epilepsy Res. 2009;83(1):1-43.

48. Stockis A, Chanteux H, Rosa M, Rolan P. Brivaracetam and carbamazepine interaction in healthy subjects and in vitro. Epilepsy Res. 2015;113:19-27.

49. Stockis A, Watanabe S, Fauchoux N. Interaction between brivaracetam $(100 \mathrm{mg} /$ day $)$ and a combination oral contraceptive: a randomized, doubleblind, placebo-controlled study. Epilepsia. 2014;55(3):e27-e31.

50. Palleria C, Coppola A, Citraro R, et al. Perspectives on treatment options for mesial temporal lobe epilepsy with hippocampal sclerosis. Expert Opin Pharma cother. 2015;16(15):2355-2371.
Neuropsychiatric Disease and Treatment

\section{Publish your work in this journal}

Neuropsychiatric Disease and Treatment is an international, peerreviewed journal of clinical therapeutics and pharmacology focusing on concise rapid reporting of clinical or pre-clinical studies on a range of neuropsychiatric and neurological disorders. This journal is indexed on PubMed Central, the 'PsycINFO' database and CAS,

\section{Dovepress}

and is the official journal of The International Neuropsychiatric Association (INA). The manuscript management system is completely online and includes a very quick and fair peer-review system, which is all easy to use. Visit http://www.dovepress.com/testimonials.php to read real quotes from published authors. 